Proceedings of the 363. WE-Heraeus Seminar on: "Neutron Stars and Pulsars" (Posters and contributed talks)

Physikzentrum Bad Honnef, Germany, May. 14 - 19, 2006, eds. W. Becker, H.H. Huang, MPE Report 291, pp. 72- 75

\title{
Giant Pulses of Pulsar Radio Emission
}

\begin{abstract}
A. D. Kuzmin
Pushchino Radio Astronomy Observatory, Lebedev Physical Institute, Russian Academy of Sciences, Pushchino, 142290, Russia e-mail: akuzmin@prao.ru
\end{abstract}

\begin{abstract}
Review report of giant pulses of pulsar radio emission, based on our detections of four new pulsars with giant pulses, and the comparative analysis of the previously known pulsars with giant pulses, including the Crab , pulsar and millisecond pulsar PSR B1937+21.
\end{abstract}

\section{Introduction}

Giant pulses (GPs)- a short duration outbursts- are a special form of pulsar radio emission.

This is a most striking phenomenon of the pulsar radio emission. For normal pulsars, the intensity of single pulses varies by no more then one order of magnitude (Hesse \& Wielebinski 1974, Ritchings 1976). GPs peak flux densities can exceed hundreds and thousands of times the peak flux density of regular pulses. GPs are the brightest sources of the radio emission observed in the Universe.

This rare phenomenon has been detected only in 11 pulsars among more than 1500 known ones. The firsts two ' of them to be discovered were Crab pulsar PSR B0531+21 (Staelin \& Reifenstein 1968; Argyle \& Gower 1972; Gower \& Argyle 1972) and the millisecond pulsar PSR B1937+21 (Wolsczcan, Cordes \& Stinebring 1984), and for a long time remain little investigated.

For over 20 years only these two pulsars were known to emit GPs. The last 5 years were marked by a systematic search of GPs that benefit a fast progress in the detection of nine new pulsar with GPs. They are PSR B182124 (Romani \& Johnston 2001), PSR B1112+50 (Ershov \& Kuzmin 2003), PSR B0540-69 (Johnston \& Romani 2003), PSR B0031-07 (Kuzmin, Ershov \& Losovsky 2004), PSR J0218+42 (Joshi et al. 2004), PSR B1957+20 (Joshi et al. 2004), PSR J1752+2359 (Ershov \& Kuzmin 2005), PSR J1823-3021A (Knight, Bailes et al. 2005) and PSR B0656+14 (Kuzmin \& Ershov 2006).

\section{Observations}

GPs are observed as a rare very strong pulses of pulsar radio emission standing out of the noise background and underlying ordinary individual pulses. An example of one

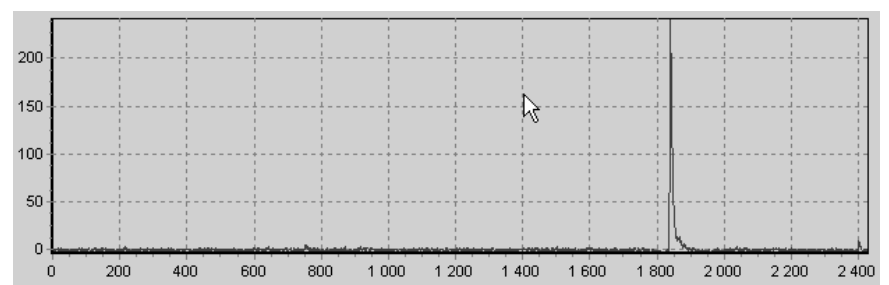

Fig. 1. An example of a GP of the Crab pulsar inside o f 150 normal pulses masked by noise

GP of the Crab pulsar observed at frequency $111 \mathrm{MHz}$ inside of 150 pulsar periods is demonstrated in Fig.1.

GPs are distinguished by a number of characteristic features. They possess very large excess of the flux density and energy of radio emission relative to an average pulse (AP), the energy distribution of GPs has a powerlaw statistic, have a short pulse time-scale and occur in a narrow-phase window of an AP (except of the Crab pulsar for which GPs can occur anywhere within the AP).

\subsection{Flux density, energy, spectra and intensity distribution.}

GPs are the most extremal phenomena of pulsar radio emission. Their peak flux densities can exceed hundreds and thousands of times the peak flux density of an AP. An example of a GP in comparison with an AP of the millisecond pulsar PSR B1937+21 is shown in Fig.2. The peak flux densitiy of GPs in this pulsar at $1650 \mathrm{MHz}$ ranges up to $65 \mathrm{kJy}$ and exceeds the peak flux density of an AP up to factor of $3 \times 10^{5}$ (Soglasnov et al. 2004). An energy of this GP $E=S_{\text {peak }} \times \tau$, where $\tau$ is the pulse duration, exceeds the energy of the AP by a factor of 60 .

The peak flux densities of GPs from the Crab Nebula pulsar PSR B0531+21 at $2228 \mathrm{MHz}$ exceeds the peak flux density of an AP up to factor of $5 \times 10^{5}$. An energy $E=$ $S_{\text {peak }} \times \tau$ of the GPs exceeds the energy of the AP by a factor of 80 (Kostyuk et al., 2003).

GPs of the Crab pulsar have been detected in a very wide frequency range from $23 \mathrm{MHz}$ (Popov et al. 2006) up to $15 \mathrm{GHz}$ (Hankins et al. 2000). Only small part of GPs 


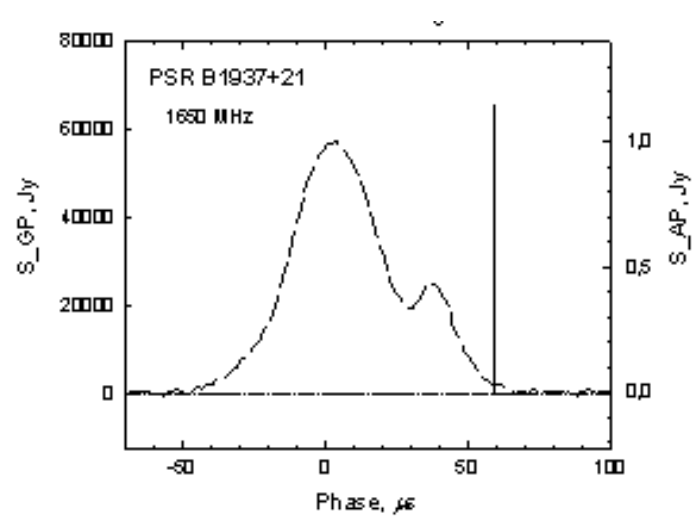

Fig. 2. An example of a GP (solid line) in comparison with an AP (dotted line) of the pulsar PSR B1937+21. (After Soglasnov et al. 2005). Note different y-scales for a GP (left) and an AP (right).

occurs simultaneously in different frequency ranges. Radio spectra of these GPs were studied by simultaneous multifrequency observations. Sallmen et al. (1999) observations of GPs in pulsar PSR B0531+21 at two frequencies 1.4 and $0.6 \mathrm{GHz}$ show that the GPs spectral indices fall between -2.2 and -4.9 , which may be compared to the AP value for this pulsar -3.0. Popov et al.(2006) observations of this pulsar at three frequencies 600,111 and $43 \mathrm{MHz}$ reveal that the GPs spectral indices fall between -1.6 and -3.1 with mean value -2.7 , that also may be compared to the AP value for this pulsar. Simultaneous two-frequency observations of GPs from PSR B1937+21 at 2210-2250 and 1414-1446 MHz (Popov \& Stappers 2003) don't reveal any GPs which occur simultaneously in both frequency ranges. They conclude that radio spectra of detected GPs are limited in frequency at a scale of about $\Delta \nu / \nu<0.5$. Simultaneous two-frequency observations of GPs in PSR B0031-07 at 111 and $40 \mathrm{MHz}$ reveal only a few common GPs (Kuzmin et al. 05).

Kinkhabwala et al. (2000) have estimated the average spectral properties of the GPs emission of the pulsar PSR B1937+21. Using the top eight GPs at three frequencies 430, 1420 and $2380 \mathrm{MHz}$, they find a somewhat steeper slope of -3.1 for the GPs spectrum, compared the -2.6 slope for the normal emission spectrum of this pulsar.

Another distinguishing characteristic of pulsars with GPs, as demonstrated in Fig.3, is their two-mode pulse intensity distribution. At low intensities of ordinary pulses, the pulse strength distribution is Gaussian one, but above a certain threshold the pulse strength of GPs is roughly power-law distributed (Argyle \& Gower 1972, Cognard et al. 1996) .

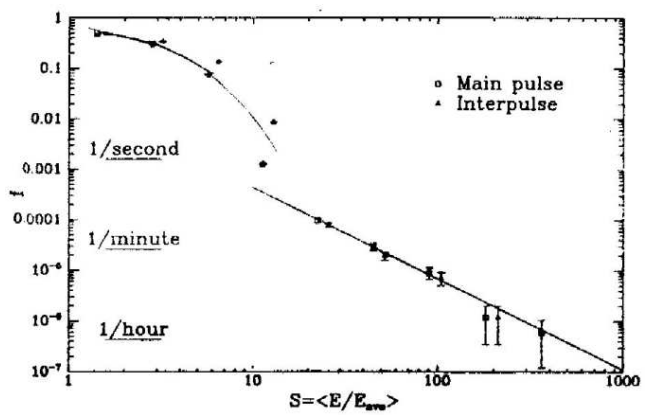

Fig. 3. a) Cumulative distribution of GPs intensity of pulsar PSR B1937+21 measured in units of the mean pulse and interpulse (after Cognars et al. 1996)

\subsection{Size and brightness temperature of an emitting region.}

The next peculiarity of GPs, as demonstrated in Fig.2 for PSR B1937+21, is a much shorter time-scale of GP as compared with an AP. The width of this GP is $\tau \leq 15$ ns (Soglasnov et al. 2004).

Hankins et al. (2003) found in pulsar PSR B0531+21 the pulse structure as short as 2 ns. If one interprets this pulse duration in terms of the maximum possible size of emitting region $l \leq c \times \tau$, where $c$ is the speed of a light, the time-scale $\tau=2$ ns corresponds to a light-travel size of an emitting body $l$ of only $60 \mathrm{~cm}$, the smallest object ever detected outside our solar system.

The brightness temperature of the GPs is

$$
T_{\mathrm{B}}=S_{\text {peak }} \lambda^{2} / 2 k \Omega,
$$

where $S_{\text {peak }}$ is the peak flux density, $\lambda$ is the radio wavelength, $k$ is the Boltzmann's constant, and $\Omega \simeq(l / d)^{2}$ is the solid angle of the radio emission region.

For observed in Crab pulsar at frequency $5.5 \mathrm{GHz}$ peak flux density of $10^{3}$ Jy with $\tau=2 \mathrm{~ns}$ (Hankins et al. 2003) the brightness temperature of GPs is as high as $10^{37} \mathrm{~K}$.

Soglasnov et al. (2004) have proved that the majority of GPs from the millisecond pulsar PSR B1937+21 are shorter than $15 \mathrm{~ns}$. At a frequency of $1.65 \mathrm{GHz}$ a peak flux density of $65 \times 10^{3}$ Jy with $\tau=15$ ns show that the brightness temperature of GPs in this pulsar is $T_{B} \geq 5 \times$ $10^{39} \mathrm{~K}$, the brightest radio emission observed in Universe.

However, these evaluations of size and brightness temperature of an emitting body are not unambiguous. Gil \& Melikidze (2004) argued that the apparent duration of the observed impulse $\tau_{\text {obs }}$ may be shorter ${ }^{1}$ ) than the duration of the emitted one as $\tau_{\text {rad }}$ as $\tau_{\text {obs }}=\tau_{\text {rad }} \times \gamma^{-2}$. For Lorentz factor $\gamma \approx 100$, the time-scale of pulse structure for B1937+21 will transformed from observed $\tau_{o b s}=2 \mathrm{~ns}$ to emitted $\tau_{\text {rad }}=20 \mu \mathrm{s}$. 1 As well as $T_{B} \propto \Omega^{-1} \propto \tau^{-2}$, the brightness temperature will be reduced to $T_{B} \approx 10^{31}$ $\mathrm{K}$.

1 Relativistic shortening of a pulse duration were claimed by Smith (1970) and Zheleznyakov (1971). 


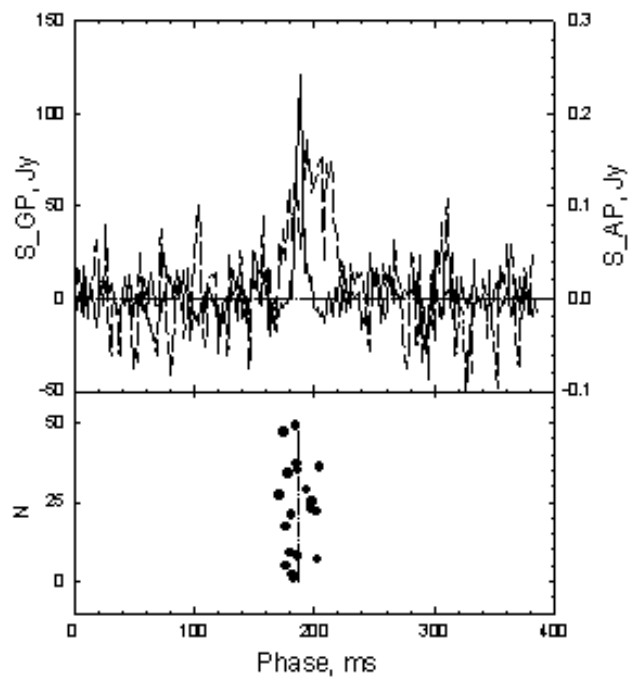

Fig. 4. (top) GP in pulsar PSR B0656+14 at $111 \mathrm{MHz}$ (bold line) together with the AP (dotted line). The plot of AP is presented on a 500 times larger scale and flux densities of GP and AP are shown separately on the left and right sides of the "y" scales. (bottom) The phases of the observed GPs relative to the center of the AP. $\mathrm{N}$ is the number of GP. (after Kuzmin \& Ershov ,2006)

This aspect needs a further refinement.

The duration of GPs may be masked by the scatter broadening of a pulse due to multi-path propagation in the interstellar plasma.

GPs are clustered around a small phase window (except of Crab pulsar, for which GPs can occur anywhere within the AP). An example of such clustering for pulsar PSR B0031-07 is shown in the bottom of Fig.4.

The top of Fig4 shows the GP together with the AP for the pulsar PSR B0656+14 (Kuzmin \& Ershov 2006). The peak flux density of this GP exceeds the peak flux density of AP by factor of 630 .

Kuzmin \& Ershov (2004) two frequency observations of GPs in the pulsar PSR B0031-07 reveal that some GPs have two components and that the spacing of these components at $40 \mathrm{MHz}$ is larger than at $111 \mathrm{MHz}$. This is similar to a larger width of the AP at lower frequency, which is interpreted as a divergence of magnetic field lines in the radius-to-frequency mapping in the hollow cone model of pulsar radio emission. This suggests that GPs from this pulsar originate in the polar region rather than in the light cylinder region.

One may suggests that there are two classes of GPs, one associated with high-energy emission from the outer gap, the other associated with the polar radio emission. GPs of PSR B0031-07 will be of the second class.

Table 1 summarizes the comparative data for known pulsars with GPs. Here PSR is a pulsar name, $P$ is the pulsar period, $B_{L C}$ is a magnetic field strength at the light cylinder, Freq is the observations frequency, $S_{G P} / S_{A P}$ is an excess of the peak flux density of a strongest GP over the peak flux density of an $\mathrm{AP}, T_{B}$ is a brightness temperature of the strongest GP radio emission and $E_{G P} / E_{G P}$ is an excess of the energy of the strongest GP over the energy of an AP.

\section{Are giant pulses inherent to some special property of pulsars?}

The first detected pulsars with GPs PSR B0531+21 and PSR B1937+21 were among a small group of pulsars with highest magnetic field at the light cylinder $B_{L C}=$ $10^{4}-10^{5} \mathrm{G}$. This gave a rise to the suggestion that GPs are inherent to pulsars with very strong magnetic field at the light cylinder and the search of GPs was oriented on those pulsars. As a result GPs were detected in five other pulsars with very strong magnetic field at the light cylinder: PSR B1821-24 (Romani \& Johnston 2001), PSR B0540-69 (Johnston \& Romani 2003), PSR J0218+42 (Joshi et al. 2004), PSR B1957+20 (Joshi et al. 2004) and PSR J18233021 (Knight et al. 2005).

However detection of the GPs in four pulsar with ordinary magnetic field at the light cylinder: PSR B003107 (Kuzmin, Ershov \& Losovsky 2004), PSR B0656+14 (Kuzmin \& Ershov 2006), PSR B1112+50 (Ershov \& Kuzmin 2003) and PSR J1752+2359 (Ershov \& Kuzmin 2005) have revealed that GPs can occur in ordinary pulsars too.

Johnson \& Romany (2004) claimed that GPs may be associated with pulsars which possess a high energy X-ray emission. But the alignment of X-ray pulses with GPs is observed only for four objects among eleven known pulsars with GPs and is not a proper indication for GPs.

Knight et all. (2005) argued that GPs may be indicated by large rotation loss luminosity $\dot{E} \propto P^{-3} \dot{P}$. But in fact the rotation loss luminosity of the known 11 pulsars with GPs are differ by 6 orders of magnitude and is not a proper indication for GPs also.

GPs occur in various types of pulsars in a wide range of periods $P=1.5-1600 \mathrm{~ms}$ and magnetic field at the light cylinder $\log B_{L C}=4-10^{6} \mathrm{G}$ over a wide range of radio frequencies.

\section{Summary}

Giant pulses is a special form of pulsar radio emission, that is characterized by very large excess of flux density and energy of radio emission relative to an average pulse, the power-law statistic of the energy distribution, giant pulses occur in a narrow-phase window of an average pulse and have a short pulse time-scale.

The flux density of giant pulses increases over the flux density of an average pulse $S_{G P} / S_{A P}$ up to $5 \times 10^{5}$.

Giant pulses energy excess over the energy of an average pulse is $E_{G P} / E_{A P}=50-200$ and is nearly the same 
Table 1. Properties of the Giant Pulses

\begin{tabular}{|c|c|c|c|c|c|c|c|}
\hline PSR & $\begin{array}{l}P \\
\mathrm{~ms}\end{array}$ & $\begin{array}{c}\log B_{L C} \\
\mathrm{G}\end{array}$ & $\begin{array}{l}\text { Freq } \\
\mathrm{GHz}\end{array}$ & $S_{G P} / S_{A P}$ & $\begin{array}{l}T_{B} \\
\mathrm{~K} \\
\end{array}$ & $E_{G P} / E_{G P}$ & References \\
\hline \multirow[t]{2}{*}{ B0031-07 } & 943 & 6.9 & 0.04 & 400 & $\geq 10^{28}$ & 75 & Kuzmin \& Ershov 2004 \\
\hline & & & 0.11 & 120 & $\geq 10^{26}$ & 8 & Kuzmin et al. 2004 \\
\hline $\mathrm{J} 0218+42$ & 2.3 & $3.2 \times 10^{5}$ & 0.61 & & $\overline{1.3}$ & 51 & Joshi et al. 2004 \\
\hline \multirow[t]{4}{*}{$\mathrm{B} 0531+21$} & 33.4 & $9.8 \times 10^{5}$ & 0.14 & 300 & & & Argyle \& Gower 1972 \\
\hline & & & 0.59 & $6 \times 10^{4}$ & $\geq 10^{36}$ & 75 & Kostyuk et al. 2003 \\
\hline & & & 2.23 & $5 \times 10^{5}$ & $\geq 10^{34}$ & 80 & Kostyuk et al. 2003 \\
\hline & & & 5.50 & & $\geq 10^{37}$ & & Hankins et al. 2003 \\
\hline B0540-69 & 50.5 & $3.5 \times 10^{5}$ & 1.38 & $5 \times 10^{3}$ & & & Johnston \& Romani 2003 \\
\hline B0656+14 & 385 & 770 & 0.11 & 600 & $\geq 10^{26}$ & 110 & Kuzmin \& Ershov 2006 \\
\hline $\mathrm{B} 1112+50$ & 1656 & 4.1 & 0.11 & 80 & $\geq 10^{26}$ & 10 & Ershov \& Kuzmin 2003 \\
\hline $\mathrm{J} 1752+2359$ & 409 & 71 & 0.11 & 260 & $\geq 10^{28}$ & 200 & Ershov \& Kuzmin 2005 \\
\hline B1821-24 & 3.0 & $7.2 \times 10^{5}$ & 1.51 & & & 81 & Romani \& Johnston 2001 \\
\hline \multirow[t]{2}{*}{ J1823-3021A } & 5.4 & $2.5 \times 10^{5}$ & 0.68 & 680 & & 64 & Knight et al. 2005 \\
\hline & & & 1.40 & 1700 & & 28 & Knight et al. 2005 \\
\hline \multirow[t]{2}{*}{ B1937+21 } & 1.5 & $9.8 \times 10^{5}$ & 0.11 & 600 & $\geq 10^{35}$ & 65 & Kuzmin \& Losovsky \\
\hline & & & 1.65 & & $\geq 5 \times 10^{39}$ & 60 & Soglasnov et al. 2004 \\
\hline $\mathrm{B} 1957+20$ & 1.6 & $3.8 \times 10^{5}$ & 0.61 & & & 129 & Joshi et al. 2004 \\
\hline
\end{tabular}

for different magnetic field at the light cylinder, pulsar periods and frequencies.

A light-travel size of an emitting body indicate the smallest object ever detected outside our solar system.

Giant pulses are the brightest sources of radio emission among the known astronomical objects.

Giant pulses exist in various types of pulsars in a wide range of periods, magnetic field at the light cylinder and broad frequency range.

Acknowledgements. This work was supported in part be the Russian Foundation of Basic Research, Project N 05-02-16415.

\section{References}

Argyle, E.,\& Gower, F.R., 1972, ApJ, 175, L89

Cognard, I., Shrauner, J.A., Taylor, J.H.,\& Thorset, S.H., 1996 ApJ. 457, L81.

Ershov, A. A.,\& Kuzmin, A. D. 2003, Pis'ma v AZh, 29, 111 (Astr. Lett., 29, 91)

Ershov, A.A.,\& Kuzmin, A.D. 2005, A\&A, 443, 593

Gil, J.,\& Melikidze, G. I. 2004, In: F. Camilo, B. M. Gaensler, eds., Young Neutron Stars and Their Environments, IAU Symposium 218, San Francisco: ASP, p. 321

Gower, F.R. \& Argyle, E. 1972, ApJ, 1715, L23

Hankins, T.H., Wex, N.\& Wielebinski, R. 2000, in Pulsar Astronomy-2000 and Beyong, ed. M. Kramer, IAU Colloquium 177, San Francisco: ASP, 202, p. 165

Hankins, T.H.,\& Kern, J.S., Weatherall, J.C.,\& Eilek, J.A. 2003, Nature, 422, 141

Hesse, T.H.,\& Wielebinski, R., 1974, A\&A, 31, 409

Johnston, S.,\& Romani, R. W. 2003, ApJ, 590, L95

Johnston, S.\& Romani, R.W. 2004, in Young Neutron Stars and Their Environments, IAU Symposium 218, ed F. Camilo, B. M. Gaensler, San Francisco: ASP, p. 315
Joshi, B. C., Kramer, M., Lyne, A.G., McLaughlin, M., \& Stairs I.H. 2004, in Young Neutron Stars and Their Environments, IAU Symposium 218, ed F. Camilo, B. M. Gaensler, San Francisco: ASP, p. 319

Kinkhabwala, A.,\& Thorset S.E. 2000, ApJ, 535,365

Knight, H. S., Bailes, M., Manchester, R. N.,\& Ord S. M. 2005, ApJ, 625, 951

Kostyuk, S.V., Kondratiev, V.I., Kuzmin, A.D., Popov, M.V., \& Soglasnov V.A. 2003, Pis'ma v AZh, 29, 440 (Astr. Lett., $29,387)$

Kuzmin, A.D.,\& Losovsky, B.Ya. 2002, Pis'ma v AZh, 28, 25 (Astr. Lett., 28, 21)

Kuzmin, A. D., Ershov, A. A.,\& Losovsky B. Ya. 2004, Pis'ma v AZh, 30, 285 (Astr. Lett., 30, 247)

Kuzmin, A. D.,\& Ershov A.A. 2004, A\&A, 427, 575

Kuzmin, A. D. \& Ershov, A. A. 2006, Pis'ma v AZh, 32, 650 (Astr. Lett., 32)

Popov, M.V.,\& Stappers, B. 2003, Pis'ma v AZh, 29, 111 (Astr. Lett., 29, 91)

Popov, M, V., Kuzmin, A. D., Ulyanov, O. M., Deshpande, A. A., Ershov, A. A., Zakharenko, V. V., Kondratiev, V. I., Kostyuk, S. V., Losovsky, B. Ya. \& Soglasnov V. A. 2006, AZh, 50, 562, (Astr. Report, 50)

Ritchings R.T. 1976, MNRAS, 176, 249

Romani, R. W., \& Johnston, S. 2001, ApJ, 557, L93

Sallmen, S., Backer D. C, Hankins, T. H., Moffet. D. \& Lundgren. S. 1999, ApJ, 517, 460.

Smith F.G. 1970, MNRAS, 149, 1

Soglasnov, V.A., Popov, M.V., Bartel N., Cannon, W., Novikov, A.Yu., Kondratiev, V.I.,\& Altunin, V.I., 2004, ApJ, 616, 439

Staelin, D. H.,\& Reifenstein E,C. 1968, Science, 162, 1481

Staelin, D. H.,\& Sutton J. M. 1970, Nature, 226, 69

Wolszczan, A., Cordes, J. M., \& Stinebring D. R. 1984, In: in Millisecond Pulsars, ed. S. P. Reynolds and D. R. Stinebring, NRAO, Green Bank, p. 63 
A. D. Kuzmin: Giant Pulses of Pulsar Radio Emission

Zheleznyakov V.V. 1971, ApSS, 13, 87 